\title{
O fisioterapeuta como profissional de suporte à parturiente
}

\author{
The physiotherapist as a professional to assist pregnant women
}

\author{
Gabriela Zanella Bavaresco ${ }^{1}$ \\ Renata Stefânia Olah de Souza ${ }^{2}$ \\ Berta Almeica ${ }^{2}$ \\ JoséH ugo Sabatino ${ }^{2}$ \\ Mirella Dias ${ }^{1}$
}

${ }^{1}$ Centro de Ciências da Saúdeedo Esporte, Universidade do Estado de SantaCatarina. Rua Pascoa Simone, 358 - Coqueiros. 88080-350 Florianópolis SC.

gabi_zanella@terra.com.br ${ }^{2}$ Grüpo de Parto Alternativo Setor deO bstetrícia, Centro deAtenção Integral à Saúde da Mulher, Universidadede Campinas.

\begin{abstract}
Control of pain during labor and childbirth are among the goals of the obstetric team to ensure the pregnant woman a safe and satisfactory delivery. There are several techniques that can be used by the physiotherapist as a member of the obstetric team to ensure relief from pain during childbirth. The physiotherapeutic assistance includes baths, cryotherapy, massage, breathing techniques, walking, vertical positions and transcutaneous electrical nerve stimulation (TENS). Bibliographical research conducted, revealed that the use of TEN S for analgesic purposes is inconclusive, though all the other techniques are considered in the literature to be beneficial, and should be encouraged during dilation and delivery. Physiotherapists proved to be useful in assisting the pregnant woman during labor, helping to reduce the perception of pain and speeding up delivery.
\end{abstract}

Key words Physiotherapy, Pregnancy, Labor, Delivery, Pain
Resumo 0 controle da dor no trabalho de parto e no parto, assim como a prevenção do sofrimento são alguns dos objetivos da equipe obstétrica, a qual deve trabalhar para garantir à mulher um parto seguro e satisfatório. Há diversos recursos que podem ser utilizados pelo fisioterapeuta enquanto membro da equipe obstétrica para proporcionar confiança, conforto e alívio da dor à parturiente durante 0 trabalho de parto. 0 suporte fisioterapêutico inclui banhos, crioterapia, massagens, técnicas respiratórias, deambulação, posições verticais e a neuroeletroestimulação transcutânea (TENS). Através da pesquisa bibliográfica realizada concluiu-se que a TENS para analgesia ainda aparece com resultados inconclusivos. Todavia, todos os outros recursos aparecem na literatura como vantajosos e que devem ser estimulados durante o período de dilatação e expulsão. 0 fisioterapeuta mostrou-se útil no acompanhamento da mulher durante o processo parturitivo, ajudando na redução da percepção dolorosa e na diminuição do tempo de trabalho de parto. Palavras-chave Fisioterapia, Gestação, TrabaIho de parto, Parto, Dor 


\section{Introdução}

A assistência profissional atual ao processo parturitivo é muitas vezes organizada em função das necessidades das instituições e não das parturientes, epor isso vem exigindo atitudes eprocedimentos que priorizem a qualidade da atenção prestada, ultrapassando o modelo de atenção centrado apenas no monitoramento e controle derisco, em favor de procedimentos preventivos em direção a saúde.

Se o processo de gestar e parir for percebido como um evento biopsicossocial, a escolha de procedimentos que permitam a participação ativa da mulher pode facilitar a promoção da saúde, a proteção ao nascimento e tornar esta expe riência muito satisfatória para a mulher ${ }^{2}$.

Há pelo menos três décadas, ocorrem esforços mundiais pela re-introdução da liberdade corporal durante o trabalho de parto, no intuito de favorecer o parto via vaginal. Em nosso país, o programa de humanização do parto e nascimento lançado em 2000 pelo M inistério da Saúde, vem tentando recuperar a participação mais ativa da gestante de baixo risco durante o processo de parturição, buscando implementar condutas baseadas em evidências científicas, incentivos ao parto vaginal, assistência menos tecnocrática eintervencionista ${ }^{3}$.

A presença do fisioterapeuta no acompanhamento do trabalho de parto não é uma prática estabelecida na nossa sociedade e nem incluída no sistema desaúde. Porém, este profissional tem a importantefunção de orientar econscientizar a mulher para que ela desenvolva toda a sua potencialidade, que será exigida neste momento, tornado-a segura e confiante 4 .

A dor no trabalho de parto e parto é um importante obstáculo que pode ser encarado e vivenciado de forma positiva pela mulher e por seus familiares. Para isto, ela necessita estar preparada e consciente da necessi dade de manter-se calma erelaxada durante todo o trabalho de parto. A utilização de métodos, que permitam vencer de maneira natural a dor, é aconselhada por muitos pesquisadores, que são unânimes em apontar os efeitos danosos que os medicamentos anal gésicos e anestésicos podem cau sar à mãe e ao feto durante o processo de parturiçãa $0^{5,6}$.

A intervenção fisioterapêutica na assistência obstétrica de baixo risco, como parteda rotina da equipe, valoriza a responsabilidade da gestante no processo, por meio do uso ativo do próprio corpo. A mobilidade corporal durante o processo departurição, envolveinteração defatores fisi- ológicos, psicológicos, culturaise, principalmente, 0 apoio ea orientação da equipe obstétrica. A ação do fisioterapeutaéum fator estimulantepara que a mulher se conscientize de que seu corpo ativo pode ser uma ferramenta para facilitar 0 processo do trabalho de parto e trazer-Ihe satisfação com a experiência do nascimento? ${ }^{7}$.

Alguns trabal hos descrevem técnicas fisioterapêuticas que podem ser aplicadas à parturiente de baixo risco para proporcionar conforto, alivio da dor, relaxamento e confiança em relação ao próprio corpo. Estímulo à deambulação, adoção de posturas verticais, exercícios respiratórios, analgesia através da neuroeletroestimulação transcutânea (TENS), massagens, banhos quentes, crioterapia erelaxamento, são exemplos dessas técnicas ${ }^{1,5-23}$.

Observou-se que as estratégias não farmacológicas no alívio da dor como os exercícios respiratórios, relaxamento muscular, massagem lombossacral e banho de chuveiro, apresentaram grande aceitação pelas gestantes com baixo risco obstétrico9.

A revisão a cerca desses recursos éimportante para que se tenha uma visão científica de seu uso, e como cada um pode influenciar a fisiologia da dor e a evolução do trabalho de parto.

\section{M etodologia}

O levantamento bibliográfico teve início com pesquisa de artigos dos últimos 05 anos (20022007) na Biblioteca Virtual em Saúde (BIREM E) que reúne bancos de dados on-line como a SciELO (Scientific Eletronic Library On Line), Lilacs (Literatura Latino-americana e do Caribe em Ciências da Saúde) e M edline ( $N$ ational Library of Medicine). Foram utilizados também materiais bibliográficos encontrados no acervo das autoras e nas bibliotecas do centro de Educação Física, Fisioterapia e D esportos do Estado deSanta Catarina - CEFID - UDESC, Biblioteca da Universidade Federal de Santa Catarina - UFSC, Biblioteca do Hospital Universitário - HU UFSC e Sistema de Bibliotecas da Unicamp.

As palavras cruzadas e empregadas para a busca dos materiais na BIREME, obtidas junto ao DeCS (Descritores em Ciências da Saúde), foram: Trabal ho de parto e Fisioterapia, Parto e Fisioterapia, Gravidez eFisioterapia, D or do Parto e Fisioterapia. Também foi utilizada a expressão "Humanização do Parto" que possui o descritor "Parto Humanizado" como sinônimo no DeCS, eque também foi cruzada com o termo "Fisiote- 
rapia". Para refinar a pesquisa, houve preferência por artigos em português.

Para a escolha das 30 referências mais relevantes para o desenvolvimento desta revisão, os materiais relacionados ao tema proposto foram selecionados com base no grau de importância e na presença palavras-chaves semelhantes às usadas na pesquisa na Bireme. Em seguida, a leitura atenta dos materiais permitiu captar as informações que respondem ao objetivo do estudo.

Estímulo à deambulação e adoção de posturas verticais

Desde a década de 70, estudos apontam para a redescoberta das posturas verticais e da mobilidade materna durante 0 trabalho de parto como prática que volta a mostrar evidências vantajosas e seguras para a parturiente $e^{6,11,24}$.

0 movimento de caminhar associado à ação da gravidade, e o aumento do canal de parto, este último proporcionado pela posição de cócoras, são considerados os principais motivos de benefícios da postura ativa da mulher durante o trabal ho de parto, pois estão ligados principalmenteà menor duração do período de dilatação, expulsivo e melhor dinâmica da contratilidade uterina. Além disso, favorecem a diminuição do uso de analgesia em mulheres que utilizaram as posturas verticais no primeiro e no segundo estágios do trabalho de parto $0^{6,7,10}$.

$\mathrm{N}$ a posição vertical há menos irregularidade na contratilidade uterina em sua forma e ritmo, podendo ser consequência da melhor irrigação sanguínea do útero nesta posição, além de pre venir a oclusão da aorta e da veia cava, consequência da postura em decúbito dorsal, assegurando assim o intercâmbio materno-fetal ediminuindo o risco de sofrimento fetal, mesmo num período expulsivo prolongado 6 .

Acredita-se que a mobilidade materna e a liberdade de movimento facilitem a "adaptação" e 0 encaixe do bebê à pelve materna e ao canal de parto, visto que a mobilidade pélvica é diferente nas diversas posturas que a mulher pode assumir durante 0 trabalho de parto ${ }^{25}$.

O utras posturas também podem ser estimuladas e auxiliadas pelo fisioterapeuta, tais como: ajoelhada, sentada, quatro apoios, sentada em cadeiras especiais, inclinada para frente apoiada em uma parede, segurando-se a uma corda presa ao teto ou a uma barra, posição de Simms (decúbito lateral esquerdo) ou cócoras sustentada, para queassim haja maior relaxamento, prin- cipalmente da musculatura dorsal, do assoalho pélvico e do canal vaginal ${ }^{5}$.

A bola suíça também pode ser usada como recurso pelo fisioterapeuta durante o primeiro estágio do trabalho de parto, com a finalidade de melhorar a percepção da tensão e do relaxamento do assoal ho pélvico da gestante, através da realização de movimentos associados à respiração, promovendo desta forma, inclusive, o relaxamento global da mulher. 0 uso da bola suíça por parturientes é um recurso a mais a ser incentivado pelos profissionais de saúde, por tratar-se de outra opção de conforto para a promoção da posição vertical durante 0 trabalho de parto ${ }^{11}$.

Em revisão sistemática de estudos sobre o posicionamento da parturiente duranteo segundo estágio do trabalho de parto, autores concluíram que o posicionamento vertical reduziu 0 tempo expulsivo, diminuiu o índice de partos instrumentalizados, de uso de ocitócitos, de episiotomia e da intensidade da dor referida no período expulsivo. Entretanto aumentou a perda sanguínea materna ${ }^{12}$.

H abitualmente a postura de cócoras não nos é solicitada e o fortalecimento do assoal ho pélvico não érotina na nossa sociedade. Em vista disso, a atuação fisioterapêutica é primordial para as parturientes queoptarem por este tipo de parto, pois através de exercícios perineais elas podem trabalhar esta musculatura a fim de prevenirem rupturas perineai $s^{5,8,12}$.

Autores sugerem que as gestantes de baixo risco deveriam ser encorajadas e estimuladas a dar à luz da maneira que se sentirem mais confortáveis, sendo informadas das vantagens e desvantagens que cada opção traz ${ }^{12,13}$.

\section{Exercícios respiratórios e relaxamento}

A respiração tem importância fundamental durante 0 trabal ho de parto e no parto, por promover o relaxamento, obter concentração, diminuir riscos de trauma perineal no momento expulsivo e melhorar a oxigenação sanguínea da mãe e do feto ${ }^{5,6}$.

Para alguns autores, não existe técnica respiratória ideal a ser recomendada durante o trabaIho de parto. 0 quedeve ser estimulado pel o fisioterapeuta, desde o pré natal, é o uso adequado dos músculos respiratórios, através da respiração espontânea, diafragmática, natural e leve, para que no momento do parto a mulher já esteja conscientizada e consiga desviar a atenção das dores e ben eficie a sua oxigenação e a do bebê $\hat{~}^{5}$. 
Pesquisadores atentam para o fato dequetécnicas respiratórias padronizadas se forem iniciadas muito cedo, podem aumentar a fadiga materna. Para isto, a parturiente deve ser estimulada a realizar respiração natural até quando sentir-se confortável ${ }^{13}$.

Estudiosos defendem o uso da respiração torácica ampla durante as contrações, pois assim a parturiente estaria aliviando a pressão do diafragma sobre o fundo uterino, mantendo a oxigenação sanguínea. Já para o intervalo entre as contrações a recomendação continua sendo a respiração abdominal ou diafragmática por ser levemente mais profunda e por promover maior relaxamento ${ }^{14}$.

Por muito tempo, a respiração rápida e superficial, conhecida como "cachorrinho", foi estimulada, mas atualmente a técnica foi abandonada, pois leva a um rápido aumento da taxa respiratória e uma redução da profundidade interferindo na fisiologia da respiração e nas trocas gasosas, podendo levar à hipocapnia, resultando em alcalose respiratória que tem como sinais e sintomas: tonturas, entorpecimento, parestesia e espasmo muscular, tensão, exaustão e ansiedade. Além de reduzir o fluxo de sangue, e conseqüentemente, o volume de oxigênio disponível tanto para a mãe quanto para o feto ${ }^{8}$.

Estudiosos afirmam que a respiração rápida e superficial, estaria indicada estritamente nos casos de períodos expulsivos muito rápidos, em que se pretende proteger o períneo do alongamento súbito e facilitar um desprendimento suave e relaxado da cabeça fetal. Os esforços expulsivos seriam intercalados por períodos de respiração ofegante, dando assim aos tecidos, tempo para rel axar, se adaptar e distender sob pressão $0^{13}$.

Outros autores analisaram a concentração plasmática do hormônio adrenocorticotrófico (indicativo de estresse orgânico) em parturientes submetidas a métodos não-farmacológicos de alívio da ansiedade e da dor no parto. Concluíram que as parturientes que utilizaram tais métodos obtiveram níveis séricos hormonais menores em todas as fases do trabalho de parto e pósparto imediato, sugerindo queas técnicasinterferiram positivamente no controle do estresse ${ }^{15}$.

Em um estudo que teve como intuito identificar os fatores que facilitam a experiência do parto, concluiu-se que os exercícios respiratórios foram os que mais apresentaram índices de predileção entre as mulheres ${ }^{16}$.

Durante o período expulsivo a utilização da respiração também é fundamental, pois se sabe que os puxos (esforços expulsivos), realizados através da prensa abdominal, se somam às contrações uterinas. Quando os puxos são espontâneos ou involuntários, a parturiente, a cada contração, consegue realizar um maior número de esforços que por serem de curta duração não produzem grande impacto cardiovascular. Já quando os puxos são dirigidos, a parturiente acaba realizando a M anobra deValsalva (aumento da pressão intra-abdominal por bloqueio da expiração e contração de abdominais) por maior período de tempo, o que por sua vez gera um grande impacto na oxigenação sanguínea e no sistema cardiovascular, prejudicando o binômio mãe-bebế.

A média de tempo dos puxos espontâneos e dirigidos é de 4-6 segundos e 10-30 segundos, respectivamente. Para os puxos espontâneos, a média depH existentena artéria umbilical éestatisticamente superior à encontrada no parto por puxos dirigidos, sendo que a exaustão e a fadiga materna também são mais comumente encontradas em mulheres que realizaram os puxos dirigidos ${ }^{13}$.

Em estudo realizado no Intermountain $\mathrm{H}$ ealth Care, (USA) foram reunidas 252 parturientes, todas submetidas à analgesia peridural e divididas em dois grupos: puxos espontâneos ou puxos dirigidos. Os autores concluíram queas parturientes que tiveram puxos espontâneos apresentaram menos sinais de fadiga e menos desacelerações cardíacas fetais. Entretanto, tiveram o período expulsivo mais prolongado ${ }^{26}$.

Além dos efeitos sobre a oxigenação sanguínea, o descontrole de forças, consequência dos puxos dirigidos e da Manobra de Valsalva durante o período expulsivo, não permite que haja o relaxamento da musculatura perineal esua distensão gradual, levando ao aumento do risco de laceração perineal ${ }^{27}$.

Outros pesquisadores afirmam que é importante que haja sincronia entre puxos, prensa abdominal e relaxamento da musculatura perineal para que ocorra uma diminuição do período expulsivo e menor chance de parto instrumentalizado ou com manobra de Kristeller (compressão manual sobre fundo uterino feita durante 0 período expulsivo do trabalho de parto, para empurrar o bebê em direção à vagina da mãe) ${ }^{4}$.

Para eles, no período expulsivo, a parturiente muitas vezes apresenta-se agitada, acabando por realizar respirações curtas e ofegantes duranteos intervalos das contrações, intensificando ainda mais o estado de ansiedade e fadiga ${ }^{4}$; portanto, é importante o fisioterapeuta estimular a realização de respirações profundas durante os inter- 
valos das contrações pois isso produz efeito calmante na parturiente.

Para os mesmos autores, é importante que haja o treinamento pré natal do período expulsivo, através de exercícios respiratórios e de prensa abdominal, para que a mulher em trabal ho de parto saiba como responder às contrações, além de ficar mais confiante em como se comportar e colaborar para o bom desenvolvimento do período expulsivo. Uma mulher desinformada e mal orientada (sem saber como se comportar) torna-se ansiosa frente a uma equipe, que tende a fazer inúmeras solicitações, num momento em que seu baixo nível de consciência a impede de compreender claramente4.

Não há dados que apóiem o direcionamento do esforço de expulsão durante o segundo estágio do trabalho de parto e algumas evidências sugerem que ela podeser prejudicial. Segundo os autores, em partos eutócitos de gestações de baixo risco, essa prática não deve ser incentivada. A recomendação genérica é permitir que a mulher faça esforços de puxo mais curtos e principalmente de forma espontânea ${ }^{13,27,28}$.

Estudos sugerem que o relaxamento associado aos exercícios respiratórios promove alívio do estresse da parturiente ao diminuir a secreção dehormônio adrenocorticotrófico ( $\mathrm{ACTH}$ ), que atua no mecanismo do estresse em resposta à dor, tanto na fase latente quanto na ativa ${ }^{15,17}$.

\section{Analgesia através de el etroestimulação} (TENS)

A Neuroel etroestimulação Transcutânea ou Transcutaneous Electrical Nerve Stimulation (TENS), éoutro método não-farmacológico para alívio da dor utilizado por fisioterapeutas em obstetrícia.

A TENS produz analgesia através da ativação dereceptores sensoriais periféricos, atuando através do fenômeno das comportas de dor, aumentando a produção de endorfinas ${ }^{29}$.

Dentre as várias opções existentes para controle da dor durante o trabal ho de parto, a TENS aparece como um recurso interessante, além de ser fácil de usar podendo ser interrompido rapidamente se necessário ${ }^{16,18}$.

Parecehaver concordância na literatura quanto à disposição dos eletrodos do TENS durantea fase de dilatação, sendo que um par de eletrodos deve ser colocado sobre a região paravertebral entre T8 a L1 eo segundo par na região sacral S1/ S4; correspondendo respectivamente, aos recep- tores nociceptivos associados ao primeiro e ao segundo estágio do trabalho de parto, sendo que os parâmetros dependem do estágio do trabalho de parto e da sensibilidade da parturiente ${ }^{18,29}$. Acredita-se que entre as contrações os parâmetros de frequência eintensidade devem ser diminuídos e aumentados durante as contraçõe ${ }^{30}$.

Existe a preocupação de que os eletrodos dispostos desta maneira e em alta intensidade pudessem, teoricamente, interferir na função cardíaca fetal, além da possibilidade de interferir também no próprio equipamento de monitoramento fetal. Porém não existem evidências cientificas a respeito dessas possibilidades ${ }^{13}$.

Segundo pesquisadores da área, existem fracas evidências científicas para o uso continuado do TENS no tratamento da dor obstétrica, porém não há sentido em descartar seu uso, atéque as discrepâncias entre a experiência clínica, a satisfação das pacientes e as evidências científicas sejam esclarecidas, visto que na prática clínica os resultados são animadores ${ }^{30}$.

A TEN S para alívio da dor no parto foi submetido a estudos mais controlados do quequaisquer uma das outras modalidades de analgesia não-farmacológica, mas os resultados dos estudos ainda não são conclusivos ${ }^{13}$.

\section{Massagens}

A massagem sempre foi um dos meios mais naturais e instintivos de aliviar a dor e o desconforto, pois reduz a ansiedade e 0 estresse, promove relaxamento muscular, diminuição da fadiga muscular, tem ação sedativa e analgésica, traz aumento da consciência corporal, produz benefícios emocionais e equilíbrio entre sistema simpático e parassimpático. Apesar de não existir um consenso relacionando riscos e benefícios da massagem durante o trabalho de parto, na prática, observa-se que essa técnica tranquiliza a parturiente, aliviando a dor e a ansiedade, e conduzindo de maneira satisfatória o trabalho de parto ${ }^{5,19}$.

Num estudo realizado em Taiwan, observouse diminuição da ansiedade e das reações de dor durante as fases latente, ativa e de transição, no grupo de mulheres que receberam massagem, servindo também como um suporte psicológico benéfico para 0 trabalho de parto ${ }^{20}$.

A massagem duranteo trabalho de parto pode ser realizada em todo o corpo desde que a parturiente sinta-se confortável para recebêla. Há várias formas de se massagear a parturiente: massagem do tecido conjuntivo nas zonas reflexas do 
baixo ventre e na região sacral; massagem leve e suave realizada com as mãos abertas de um lado ao outro na região do baixo ventre; massagem através de batidas leves com os dedos no baixo ventre de um lado para o outro; deslizamento da região sacrococcígea até as cristas ilíacas; massagem profunda sobre a região sacral; massagem com as duas mãos sobre as articulações sacroilíacas, no sentido longitudinal do occipital até 0 cóccix, paralelamente à coluna vertebral ${ }^{5}$.

\section{Banhos quentes e crioterapia}

Durante o trabalho de parto, a água morna reduz a sensibilidade dolorosa da parturiente com re dução da atividade simpática através da modificação da transmissão aferente nociceptiva, a qual setorna mais lenta e eleva os níveis deencefalinas eendorfinas endógenas. A utilização do chuveiro, deixando a água cair sobre as costas da gestante, alivia a dor lombar, queixa presente em um terço das parturientes no primeiro estágio do parto, geralmente em consequência da apresentação posterior da cabeçado feto (ODP), provavelmente devido à demora na rotação do pólo cefálico ${ }^{21}$.

Num ensaio-clínico realizado numa maternidade pública da cidade de São Paulo, com o objetivo de identificar a influência do banho de imersão na duração do primeiro período clínico do trabalho de parto e na frequência e duração das contrações uterinas, observou-se que o banho de imersão é uma opção viável para o conforto da parturiente, pois, apresenta as vantagens de reduzir e de postergar o uso de fármacos no controle da dor, proporcionando condições para a colaboração ativa da parturiente e permitindo maior participação do acompanhante ${ }^{22}$.

0 uso do gelo é uma das mais antigas formas de analgesia por diminuir a via nervosa aferente nociceptiva por redução metabólica e isquemia da vasa nervorum e nervi nervorum, em função da intensa vasoconstrição. Além dos banhos quentes, as parturientes podem usar compressas frias ou pacote de gel congelado para reduzir a temperatura e a dor. Aplicando-se de forma correta para evitar a formação de queimaduras, a crioterapiaéuma técnica inócua e reconfortante, não impedindo o uso associado de técnicas anal gésicas mais eficazes ${ }^{21}$.

Num estudo, realizado no Hospital M aternidade Carmela Dutra, no Rio de Janeiro, usou-se gelo como recurso para alívio da dor eforam verificados possíveis efeitos sobre a evolução do trabaIho de parto e perfil biofísico do feto. Concluiu-se que a crioterapia pode ser aplicada para alívio da dor no parto, pois proporciona o relaxamento geral das parturientes, sem gerar quaisquer efeitos indesejados sobre o perfil biofísico do feto ${ }^{23}$.

\section{Conclusão}

O suporte físico eemocional promovido pelo fisioterapeuta duranteo trabal ho departo eo parto de baixo risco parecem contribuir para sua humanização ea do nascimento ao proporcionar à parturiente bem-estar físico, redução das percepções dolorosas, aumento da confiança, redução do medo eda ansiedade, emaior consciência do processo parturitivo.

Além disso, a assistência fisioterapêutica ajuda ainda a tornar o processo de parturição mais ativo, natural e satisfatório, favorecendo uma vivência positiva na vida social efamiliar da parturiente, como mulher e mãe.

Acreditamos que o fisioterapeuta é o profissional da saúde que dispõe de todo o conhecimento para fornecer este suporte de forma eficiente e segura, priorizando métodos não-farmacológicos de alívio da dor no trabalho de parto e no parto.

Porém, como a grande maioria dos hospitais ematernidades não oferece atendimento fisioterapêutico às suas gestantes, ainda temos um longo caminho a percorrer até que todas elas tenham acesso a um atendimento obstétrico mais acolhedor ehumanizado, quesupra todas as suas necessidades, respeite a sua individualidade e garanta satisfação para a família que acaba de aumentar. 


\section{Colaboradores}

RSO Souza e GZ Bavaresco foram responsáveis pelas ideias, delineamento, revisão bibliográfica, análise e redação do artigo. B Almeida, M Dias e JH Sabatino foram responsáveis pela aprovação do texto final a ser publicado.

\section{Agradecimento}

À Prof. M s. FrancineFischer-Sgrott pelo incentivo e colaboração.

\section{Referências}

1. Bio ER. Assistência fisioterapêutica na assistência ao trabalho de parto [dissertação]. São Paulo: Faculdade de M edicina da Universidade de São Paulo [dissertação na Internet]. 2007 [acessado 2008 jun 2]. Disponível em: http://www.teses.usp.br/teses/disponiveis/5/ 5139/tde-12022008-141747/

2. Domingues RM SM, Santos EM, Leal MC. Aspectos da satisfação das mulheres com a assistência ao parto: contribuição para o debate. Cad Saude Publica [periódico na Internet]. 2004 [acessado 2008 jun 2]; 20(Suppl. 1). Disponível em: http://www.scielosp.org/ scielo.php?script=sci_arttext $\&$ pid $=\$ 0102-311 \times 200$ 4000700006\&lng=en. doi: 10.1590/S0102-311X2004000 700006.

3. Serruya SJ, Lago TG, Cecatti JG. O panorama da atenção pré-natal no Brasil e o Programa de Humanização do Prénatal e Nascimento. Rev Bras Saude M ater Infant [periódico na Internet]. 2004 [acessado 2008 jun 2]; 4(3):269-279. Disponível em: http:// www.scielo.br/scielo.php?script=sci_arttext\&pid= S1519-38292004000300007\&lng=en. doi: 10.1590/S15 19-38292004000300007.

4. Cassol EGM, Canfield JT, Morais EN. Desempenho motor e emocional de parturientes durante 0 2 - período do trabalho de parto. Rev Bras Fisioter 2001; 5(1):9-16.

5. Balaskas J. Parto ativo: guia prático para o parto natural. São Paulo: Ground; 1993.

6. Sabatino H, Dunn PM, Barcia RC. Parto Humanizado: formas alternativas. Campinas: Editora da Unicamp; 2000.

7. Bio ER, Bittar RE, Zugaib M. Influência da Mobilidade $M$ aterna na duração da fase ativa do trabalho de parto. Rev Bras Ginecol Obstet [periódico na Internet]. 2006 [acessado 2008 jun 2]; 28(11):671-679. Disponível em: http://www.scielo.br/scielo.php? script=sci_arttext $\&$ pid $=\$ 0100-72032006001100007 \&$ Ing=en. doi: 10.1590/S0100-72032006001100007.

8. Calais-German B. O Períneo Feminino e o Parto: elementos de anatomia e exercícios práticos. São PauIo: M anole; 2005.

9. Davim RMB, Torres GV, M elo ES. Estratégias não farmacológicas no alívio da dor durante o trabalho de parto: pré-teste de um instrumento. Rev Lat Am Enfermagem [periódico na Internet]. 2007 dez [acessado 2008 jun 2]; 15(6):1150-1156. Disponível em: http://www.scielo.br/scielo.php?script=sci_arttext\& pid=S0104-11692007000600015\&Ing=en. doi: $10.1590 /$ S0104-11692007000600015.

10. M amede FV, Almeida AM, Clapis MJ. M ovimentação/deambulação no trabalho de parto: uma revisão. Acta Sci Health Sci [periódico na Internet]. 2004 [acessado 2008 jun 3]; 26(2):295-302. Disponível em: http://www.periodicos.uem.br/ojs/index. php/ActaSciH ealthSci/article/view/1580/932

11. Lopes TC, Madeira LM, Coelho S. O uso da bola do nascimento na promoção da posição vertical em primíparas durante o trabalho de parto. Rev M in Enferm [periódico na Internet]. 2003 [acessado 2008 jun 3]; 7(2):134-139. Disponível em: http://www. enfermagem.ufmg.br/reme/remev7n2.pdf 
12. Gupta JK, Hofmeyr G], Smyth R. Position in the se cond stage of labour for women without epidural anaesthesia. Cochrane Database of Systematic Reviews [pe ríodico na Internet]. 2007 [acessado 2008 jun 3]; 4. Disponível em: http://www.who.int/rhl/reviews/ CD 002006.pdf. doi: 10.1002/14651858.CD 002006.pub2

13. Enkin M, Keirse MJC, Neilson JP, Crowther C, Duley L, Hodnett E. Guia para a atenção efetiva na gravidez e no parto. Rio de Janeiro: Guanabara Koogan; 2005

14. Reberte LM, Hoga LAK. O desenvolvimento de um grupo de gestantes com a utilização da abordagem corporal. Texto contexto - enferm. [periódico na Internet]. 2005 [acessado 2008 jun 3]; 14(2):186-192. Disponível em: http://www.scielo.br/scielo.php? script=sci_arttext\&pid=S0104-07072005000200005\& Ing=pt. doi: 10.1590/S0104-07072005000200005.

15. Almeida NAM, Silveira NA, Bachion M M, Sousa JT. Concentração plasmática do hormônio adrenocorticotrófico de parturientes submetidas a método não farmacológico de alívio da ansiedade e dor no parto. Rev Lat Am Enfermagem [periódico na Internet]. 2005 [acessado 2008 jun 4]; 13(2):223-228. Disponível em: http://www.scielo.br/scielo.php? script=sci arttext\&pid=S0104-11692005000200014\& Ing=en. doi 10.1590/S0104-11692005000200014.

16. Sabatino H. Parto na vertical. RBM - GO 1997; 8(2): 51-64.

17. Almeida NAM, Sousa JT, Bachion M M, Silveira NA Utilização de técnicas de respiração e relaxamento para alívio de dor e ansiedade no processo de parturição. Rev Lat Am Enfermagem [periódico na Internet]. 2005 [acessado 2008 jun 4]; 13(1):52-58. Disponível em: http://www.scielo.br/scielo.php?script= sci_arttext\&pid=S0104-11692005000100009\& Ing=en . doi: 10.1590/S0104-11692005000100009.

18. Orange FA, Amorim MMR, Lima L. Uso da eletroestimulação transcutânea para alívio da dor durante 0 trabalho de parto em uma maternidade-escola: ensaio clínico controlado. Rev Bras Ginecol Obstet [periódico na Internet]. 2003 [acessado 2008 jun 4]; 25(1):45-52. Disponível em: http://www.scielo.br/ scielo.php?script $=$ sci_arttext $\&$ pid $=\$ 0100-7203200$ $3000100007 \&$ Ing=en. doi: 10.1590/S0100-7203200 3000100007.

19. Simkin PP, O'hara M. Nonpharmacologic relief of pain during labor: systematic reviews of five methods. Am J Obstet Gynecol. 2002; 186(Suppl. 5):131-159.

20. Chang MY, Wang SY, Chen CH. Effects of massage on pain and anxiety during labour: a randomized controlled trial in Taiwan. J Adv Nurs. 2002; 38(1): 68-73.

21. Vale NB. Analgesia Adjuvante e Alternativa. Rev Bras Anestesiol [periódico na Internet]. 2006 [acessado 2008 jun 4]; 56(5):530-555. Disponível em: http:// www.scielo.br/scielo.php?script=sci_arttext\&pid $=$ S0034-70942006000500012\&lng=en. doi: 10.1590/ S0034-70942006000500012.
22. Silva FM B, Oliveira SMJV. O efeito do banho de imersão na duração do trabalho de parto. Rev Esc Enferm USP [periódico na internet]. 2006 [acessado 2008 jun 4]; 40(1):57-63. Disponível em: http://www. scielo.br/scielo.php?script=sci_arttext\& $\mathrm{pid}=\mathrm{S0080}$ $62342006000100008 \&$ Ing=en. doi: 10.1590/S008062342006000100008.

23. Nunes $S$, Vargens OMC. A crioterapia como estraté gia para alívio da dor no trabalho de parto: um estudo exploratório. Rev Esc Enferm USP [periódico na Internet]. 2007 [acessado 2008 jun 5]; 15(3):337342. Disponível em: http://www.portalbvsenf.eerp. usp.br/scielo.php?script=sci_arttext\&pid=S0104-355 22007000300003\&Ing =pt\&nrm=iso. ISSN 0104-3552

24. Rooks JP. Evidence-based practice an its application to childbirth care for low-risk women. J Nurse Midwifery 1999; 44(4):337-340.

25. Simkin P. Maternal Position and Pelves Revisited. Birth 2003; 30(2):130-132.

26. Hansen SL, Clarck SL, Foster JC. Active pushing versus passive fetal descent in the second stage of labor: a randomized controlled trial. Obstet Gynecol. 2002; 99(1):29-34.

27. Grosse, D; Segler J. Reeducação Perineal. São Paulo, SP: M anole; 2002.

28. Cecatti JG, Calderón IMP. Intervenções benéficas durante o parto para a prevenção da mortalidade materna. Rev Bras Ginecol Obstet [periódico na Internet]. 2005 [acessado 2008 jun 5]; 27(6):357-65. Disponível em: http://www.scielo.br/scielo.php? script=sci_arttext $\&$ pid $=$ S0100-72032005000600011\& Ing=en. doi: 10.1590/S0100-72032005000600011.

29. Knobel R. Técnicas de acupuntura para alivio da dor no trabalho de parto - ensaio clínico [tese]. Campinas: U nicamp [tese na internet]. 2002 [acessado 2008 jun 5]. Disponível em: http://libdigi.unicamp. $\mathrm{br} / \mathrm{document} /$ ?code $=\mathrm{vt}$ |s000265427

30. Kitchen S. Eletroterapia Baseada em Evidências. 11a ed. São Paulo: Manole; 2003.

Artigo apresentado em 18/04/2009

Aprovado em 20/10/2009

Versão final apresentada em 07/12/2009 